\title{
Vertical and horizontal integration of multi- omics data with miodin
}

\author{
Benjamin Ulfenborg(1)
}

\begin{abstract}
Background: Studies on multiple modalities of omics data such as transcriptomics, genomics and proteomics are growing in popularity, since they allow us to investigate complex mechanisms across molecular layers. It is widely recognized that integrative omics analysis holds the promise to unlock novel and actionable biological insights into health and disease. Integration of multi-omics data remains challenging, however, and requires combination of several software tools and extensive technical expertise to account for the properties of heterogeneous data.

Results: This paper presents the miodin $\mathrm{R}$ package, which provides a streamlined workflow-based syntax for multiomics data analysis. The package allows users to perform analysis of omics data either across experiments on the same samples (vertical integration), or across studies on the same variables (horizontal integration). Workflows have been designed to promote transparent data analysis and reduce the technical expertise required to perform lowlevel data import and processing.

Conclusions: The miodin package is implemented in $\mathrm{R}$ and is freely available for use and extension under the GPL3 license. Package source, reference documentation and user manual are available at https://gitlab.com/algoromics/ miodin.
\end{abstract}

Keywords: Multi-omics, Data analysis, Data integration, Transparency

\section{Background}

With the advances in high-throughput biotechnology over the past two decades, we now have access to an unprecedented wealth of data for many omics modalities. In this era of biomedical big data, the primary research challenges are how to integrate and analyze large-scale data of different types and sources to gain new insights into the complex mechanisms behind health and disease [1-4]. In a study by Woo et al., DNA copy-number variation, methylation and gene expression were profiled in a cohort of hepatocellular carcinoma (HCC) patients. Integrative omics analysis revealed three molecular subtypes of HCC with differences in prognostic outcomes [5]. Zhu et al. performed a comprehensive pan-cancer integrative analysis showing that a combination of clinical variables with molecular profiles improved prognostic power in 7 of the 14 cancer types studied [6]. Lau et al. carried out a cardiac hypertrophy study in mice based on transcriptomics, proteomics and protein turnover data. The combination of

Correspondence: benjamin.ulfenborg@his.se

School of Bioscience, University of Skövde, Skövde, Sweden multi-omics data revealed complementary insights into the pathogenesis of the disease [7]. These and other studies show that the integrative approach deliver novel biological insights. Advanced bioinformatics tools and algorithms have been developed that can analyze multiple modalities of omics data [8-10], but performing transparent and reproducible integrative analysis remains a significant challenge. Notably, considerable technical expertise is required to use many tools and combine them into a coherent pipeline.

Bioconductor is one of the largest open source projects for analysis of omics data [11], hosting more than 1600 software packages as of release 3.8. Many experimental techniques (e.g. microarrays, sequencing and mass spectrometry) and omics data types (e.g. genomics, transcriptomics and proteomics) are supported [12-20]. To perform data analysis, the project hosts many packages for different workflow steps, such has import, annotation, pre-processing, quality control, statistical analysis, biological interpretation and visualization [12, 21-26]. By promoting a common set of data structures, package interoperability, version control, extensive documentation

(c) The Author(s). 2019 Open Access This article is distributed under the terms of the Creative Commons Attribution 4.0 International License (http://creativecommons.org/licenses/by/4.0/), which permits unrestricted use, distribution, and 
and high development standards, the project contributes significantly to distributing $\mathrm{R}$ software in bioinformatics. Furthermore, Bioconductor hosts experimental data, workflows, tutorials and other materials to facilitate learning, usage and combination of packages. With its large and active community, Bioconductor continues to expand to meet the future challenges in multi-omics data analysis.

Given the functionality it provides, Bioconductor is an obvious choice when selecting software for performing integrative multi-omics data analysis. However, even for seasoned bioinformaticians a lot of technical expertise and work is required to combine packages into coherent pipelines. Knowing which packages to use is an issue when working with new techniques and data, since there are several possible packages available for a given problem. Learning how to use several packages takes time, given the need to be familiar with the logic behind data structures along with multiple functions and their parameters. Another challenge is the growth in complexity of the analysis scripts, where code is required to perform every analysis step, including import, pre-processing, quality control, statistical analysis and interpretation. This problem is exacerbated when working with multiomics data and performing integrated analysis, where several steps are needed for every experimental technique and data type. This increases the risk of clerical errors and results in low transparency in terms of what processing and analysis steps that have been performed.

A related problem in omics data analysis is the lack of a systematic way to specify generic study designs in analysis scripts. Issues may include what experimental variables to analyze, how to define sample groups and statistical comparisons, how samples are paired, how to correct for batch effects and how to collapse replicates by mean. This can be performed ad hoc with e.g. variables and indexing operations, but this is error prone and gives low transparency when dealing with large datasets, multiple data types and more complex designs. Another general problem is the reproducibility of bioinformatics workflows $[27,28]$, i.e. to ensure that the same results are obtained when running a workflow on the same data with the same steps and parameters. This has been addressed by Nextflow [29] and related software, which are used to construct workflows and support Docker technology [30] for deployment. This technology ensures that the analysis environment remains the same and protects against numeric instability across different systems. The BiocImageBuilder [31] is a tool designed to promote reproducibility of Bioconductor workflows by building a Docker image configured with all necessary software. The image also supports JupyterHub [32] and Binder [33] for private and public deployment of Jupyter notebooks for sharing and rerunning the analysis.
Several tools and techniques have been developed for integrative analysis of multi-omics data [34, 35]. One popular approach is matrix factorization, where the objective is to infer latent factors that explain interpatient variance within and across omics modalities. MultiOmics Factor Analysis (MOFA) is an unsupervised matrix factorization technique that is a generalization of Principle Component Analysis to several data matrices. Two strengths of MOFA are that it can integrate data from different distributions and handle missing data [36]. The mixOmics package [37] provides both unsupervised and supervised methods based on Partial Least Squares and Canonical Correlation Analysis, with generalizations to multi-block data. Another powerful approach to data integration is graph-based clustering of samples, which has been applied to disease subtype discovery. In Similarity Network Fusion, single-omics patient similarity networks are constructed, followed by iterative exchange of information to generate a fused multi-omics patient network [38]. Another technique relies on permutation-based clustering and was developed to identify robust patient partitions. This method integrates data by detecting the agreement between omicsspecific patient connectivity [39].

To address the challenges of multi-omics data analysis, the miodin (MultI-Omics Data INtegration) $\mathrm{R}$ package was developed. The package provides a software infrastructure to build data analysis workflows that import, process and analyze multi-omics data. Workflows accommodate data from different omics modalities, including transcriptomics, genomics, epigenomics and proteomics, and from different experimental techniques (microarrays, sequencing and mass spectrometry). The package allows users to integrate omics data from different experiments on the same samples (vertical integration) or across studies on the same variables (horizontal integration). Furthermore, the user is provided with an expressive vocabulary for declaring the experimental study design, to render this explicit within the analysis script and reduce the risk of clerical errors. A key design goal when developing miodin was to streamline data analysis, by providing a clean syntax for building workflows and minimizing the extent of technical expertise required to combine multiple software packages. The motivation behind this was to promote transparent biomedical data science.

\section{Implementation}

\section{Package overview}

The miodin package was implemented following the S4 object-oriented programming paradigm. Infrastructure functionality is supported by 16 S4 classes for which a common set of standard generics (base API) has been defined. Apart from the base classes, a number of workflow module classes have been developed, which serve as the building blocks of 
workflows. On top of the base API is a high-level user API consisting of convenience operators + and $\%>\%$ along with helper functions to simply manipulation of objects (Fig. 1). The user API has been developed to reduce the learning curve for the package and minimize the number of classes, functions and parameters the user needs to learn.

Data analysis with miodin follows an intuitive three-step process illustrated in Fig. 2. The user first initializes a project, a study and a workflow. The project serves as a placeholder for all other objects and the study is used to declare the study design, including what samples and assays to analyze, and the experimental variables of interest, if any. The miodin package implements an expressive study design vocabulary and several convenience functions for common designs, such as casecontrol and time series experiments. These allow the user to declare all information required for data analysis in one place, thus reducing the risk of clerical errors in the analysis script and the amount of information the user must provide during the analysis itself. The workflow is used to build the data analysis procedure as a set of sequentially connected workflow modules that carry out specific tasks, such as data import or processing. The analysis is performed by executing the workflow, which generates datasets and results. These can be inspected, exported and used for further analysis.

\section{Study design vocabulary}

Information related to a study is managed using the MiodinStudy class. The study design can be declared manually by instantiating an empty study and using helper functions that add different properties to the design (Table 1) or using convenience functions available for some of the most common designs (Table 2).

The purpose of declaring the study design is for the user to give an explicit definition on what samples are included (in a sample table), what assays or experimental data files to analyze (in an assay table), what sample groups exist and which groups to compare during the analysis. The benefits of this are that the user can provide all this information in one place in the analysis script and that no variable manipulation is needed on the user's part. Furthermore, when the user adds sample and assay tables these are automatically validated against the declared study design to detect potential clerical errors that might otherwise disturb the downstream analysis. For detailed examples how to declare the study design, see the miodin user manual in the GitLab repository (https://gitlab.com/algoromics/miodin).

\section{Workflow syntax}

When the study design has been declared the next step is to define the data analysis workflow. A workflow is built by instantiating the MiodinWorkflow class and adding workflow modules to it, each one performing a specific task. Workflow modules are added to the workflow object by + operator and a module-specific instantiation function. To feed the output from one module as

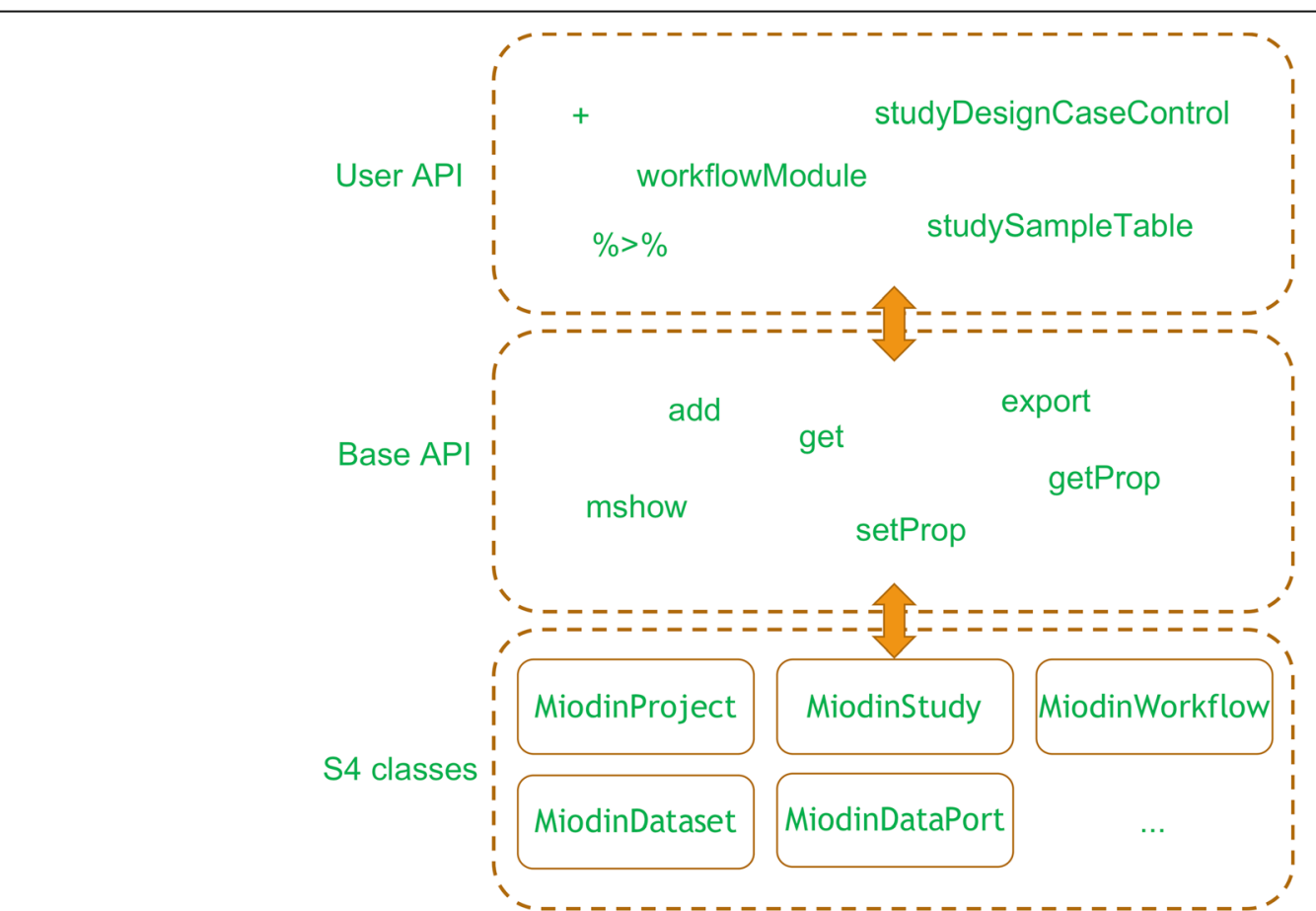

Fig. 1 Package design. The miodin package provides a software infrastructure for data analysis implemented as a set of S4 classes. The base API contains standard generics for object manipulation and the user API provides convenience functions to facilitate package usage 


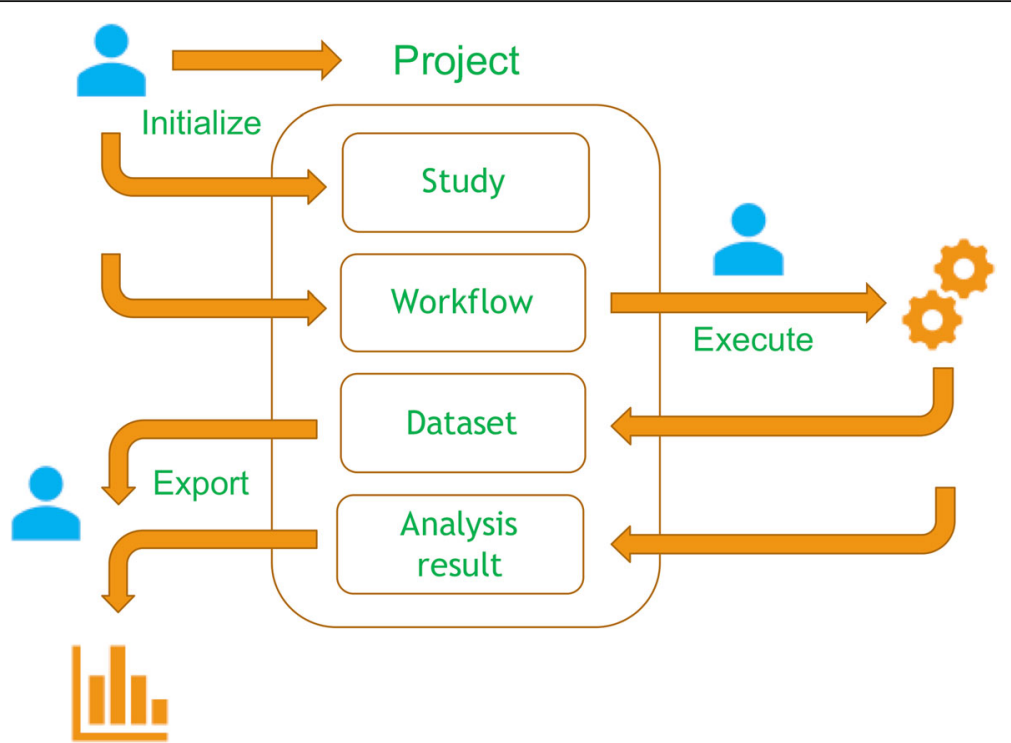

Fig. 2 Data analysis in miodin. The user starts by defining a project, a study and a workflow. The study contains the design of the experiment and the workflow is defined by instantiating analysis modules, which generate datasets and analysis results upon execution. The user can then inspect and export the data and results

input to the next, they can be combined using the pipe operator $\%>\%$.

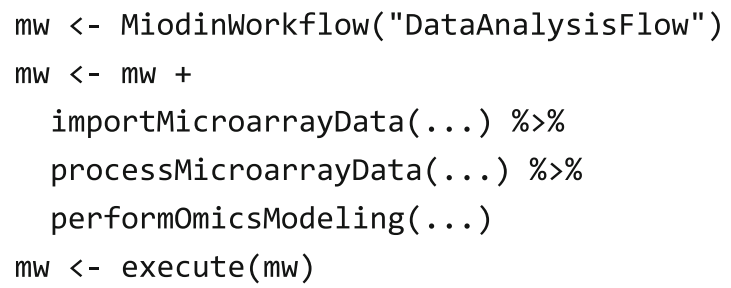

This script initializes a workflow called DataAnalysisFlow with three workflow modules. Module parameters have been omitted for brevity. The first module imports microarray data, the second processes the output from the first module, and the final module performs statistical testing on the processed data.

The analysis is carried out by calling the execute method. This syntax allows the user to define streamlined data analysis workflows, enhancing readability of the analysis script compared to longer chunks of code. Workflow modules have names starting with verbs denoting their

Table 1 Study design helper functions

\begin{tabular}{ll}
\hline Function & Description \\
\hline studySamplingPoints & Set the sampling points (e.g. time points) \\
studyFactor & Define a factor (experimental variable) \\
studyGroup & Define a sample group based on existing factors \\
studyContrast & Define a contrast (sample group comparison) \\
studySampleTable & Add a table with sample annotation data \\
studyAssayTable & Add a table with assay annotation data \\
\hline
\end{tabular}

function, making them easier to remember and improving analysis transparency. To further improve transparency, the analysis workflow automatically documents each processing and analysis step, including a description of what was done, what function was called, the name and version of the package, names and values of parameters, and how this affected the data. These can be inspected and exported as part of the dataset, thus addressing the issues of provenance [27], which is one important aspect of reproducibility.

\section{Package features}

The workflow modules available as of miodin version 0.4.1 are described in Table 3. Import, processing and analysis of data is supported for different experimental techniques and omics data types as given in Table 4. For microarrays, raw data from Affymetrix arrays (CEL format) and Illumina arrays (IDAT format) is supported, including transcriptomics, genomics (SNP) and methylation data. Processed data is also supported for microarrays, sequencing (RNA-seq counts) and mass spectrometry (protein quantification). Workflow modules store data in instances of the MiodinDataset class, which inherits from MultiDataSet [40]. The MultiDataSet class provides functions to combine data from different omics-specific objects (e.g. ExpressionSet and SummarizedExperiment) and recover the original objects later on. MiodinDataset includes additional slots to hold processed data, interactions, quality control reports, processing protocols and auxiliary data. Table 5 lists the $\mathrm{R}$ package dependencies of miodin used for bioinformatics analysis. 
Table 2 Common study design functions

\begin{tabular}{ll}
\hline Function & Description \\
\hline studyDesignCaseControl & Single factor dividing samples into two groups \\
studyDesignMultipleGroups & Single factor dividing samples into multiple groups \\
studyDesignRepeatedMeasures & Single factor and multiple sampling points \\
studyDesignTwoFactors & Two factors and multiple sampling points \\
\hline
\end{tabular}

\section{Omics data integration}

The miodin package can be used for analysis single-omics data, though by design the package is intended to streamline multi-omics data integration and analysis. Two case studies were carried out to illustrate how horizontal integration (across studies) and vertical integration (across omics modalities) can be performed. For demonstration purposes, data used in the case studies were pre-processed and included in the companion package miodindata prior to analysis with miodin. For horizontal integration, three lung cancer transcriptomics datasets with accession number E-GEOD-27262 [41], E-GEOD-19188 [42] and EGEOD-40791 [43] were downloaded from ArrrayExpress [44]. Probes were mapped to genes with NetAffx file HGU133-Plus-2-NA36 and each dataset was filtered to include only the first 2000 genes. Vertical integration was carried out using breast cancer data from the curatedTCGAData package [45]. RNA-seq gene and miRNA count data as well as $450 \mathrm{k}$ methylation data were included in the analysis. RNA-seq gene and methylation data were filtered to include only the 5000 top-variance features.

\section{Results}

Horizontal integration: meta-analysis on lung cancer transcriptomics data

To perform meta-analysis in miodin, a study design must be declared for every dataset included in the analysis. This implies defining a sample table and assay table (as data frames) and calling the appropriate study design function.
The three transcriptomics datasets used here (referred to as Wei2012, Hou2010 and Zhang2012) have case-control designs and were declared using studyDesignCaseControl (see Additional file 1). The Wei2012 dataset contained 50 samples; 25 from stage 1 lung adenocarcinoma tissue and 25 paired samples from adjacent normal tissue [41]. Sample pairedness was specified with the paired argument to the study design function, naming a column in the sample table containing information of how samples are paired. The Hou2010 dataset contained 156 samples (91 tumors and 65 healthy controls) and Zhang2012 contained 194 samples (94 tumors and 100 healthy controls).

When the study design had been declared, a workflow was built to import and process transcriptomics data. The workflow imported data from the miodindata package using importProcessedData followed by processMicroarrayData to remove genes with an expression below 5 in all samples. The three datasets were integrated using integrateAssays and linear modeling with limma [19] was carried out with performOmicsModeling. This module identifies differentially expressed genes in each individual dataset and by setting metaAnalysis to TRUE an additional step is performed to reveal concordant results between the datasets. The final results are stored as a Venn diagram accompanied by data frames, which can be exported to disk (data frames are exported as Excel sheets). The Venn diagram is shown in Fig. 3 and the list of differentially expressed genes is provided in Additional file 2. The analysis script is available in Additional file 1.

Table 3 Workflow modules

\begin{tabular}{ll}
\hline Function & Description \\
\hline downloadRepositoryData & Downloads data from an online repository \\
importMicroarrayData & Imports raw microarray data from Affymetrix and Illumina arrays \\
importProcessedData & Imports processed RNA, SNP, methylation and protein data \\
processMicroarrayData & Pre-processes microarray data \\
processSequencingData & Pre-processes sequencing data \\
processMassSpecData & Pre-processes mass spectrometry data \\
integrateAssays & Integrates several datasets into one \\
performFactorAnalysis & Performs factor analysis by matrix factorization \\
performHypothesisTest & Performs hypothesis testing \\
performLinearModeling & Performs generalized linear modeling with snpStats \\
performOmicsModeling & Performs modeling with limma, DMRcate or edgeR depending on the input object \\
\hline
\end{tabular}


Table 4 Supported experimental techniques and data types

\begin{tabular}{llll}
\hline & RNA & SNP & Methylation \\
\hline Microarray & Raw and processed & Raw and processed & Raw and processed \\
Sequencing & Processed & & \\
Mass spectrometry & & & Processed \\
\hline
\end{tabular}

\section{Vertical integration: exploratory data analysis on multi- omics data}

The TCGA data used for vertical integration contained 338 breast cancer samples, for which survival status (alive or diseased) was available. To perform vertical integration, a case-control study design was declared and one assay table for each omics modality was added, specifying the data files to import. A workflow was built to import data with importProcessedData, followed processSequencingData to perform RNA-seq count filtering and variance stabilization with DESeq2 [46]. Methylation data was processed with processMicroarrayData to remove probes flagged as problematic by DMRcate [47] and non-CpG probes. The multi-omics data was integrated with integrateAssays and integrative analysis carried out by fitting a MOFA model to the data with performFactorAnalysis. The analysis script is available in Additional file 3.

Table 5 Package dependencies

\begin{tabular}{ll}
\hline Package & Description \\
\hline AffyCompatible & Annotation of Affymetrix microarrays \\
ArrayExpress & Access to the ArrayExpress online repository \\
crlmm & Genotyping of microarray SNP data \\
DESeq2 & Processing of RNA-seq data \\
DMRcate & Statistical analysis of methylation data \\
edgeR & Statistical analysis of RNA-seq data \\
ff & Store large in-memory datasets on disk \\
limma & Statistical analysis of microarray RNA data \\
minfi & Import and normalization of microarray \\
mixOmics & methylation data \\
Methods for integrative analysis of multi-omics \\
SumpStats
\end{tabular}

The results from performFactorAnalysis include the fitted model object and plots to assess the model in terms of variance explained, sample clustering (Fig. 4) and the top features in the first factor (Fig. 5). Plots for other factors can be rendered and further downstream analysis carried out with the model object. Jupyter notebooks for reproducing the horizontal and vertical analyses are provided in GitLab (https://gitlab.com/algoromics/miodinnotebooks), with the option to run on Binder [33].

\section{Discussion}

Multi-omics experiments are indispensable for studying biological systems across molecular layers. In order to capitalize on the availability of high-throughput data and perform integrative analyses, analysts need to develop complex pipelines that can incorporate methods for import, processing and integration of different data types. Thanks to the rapid development of new bioinformatics tools, a large number of methods and software packages exist for various analysis problems. The difficulty lies in constructing a workflow that ensures transparency, scalability, reproducibility and tracking of data provenance during the analysis. A transparent workflow should reveal what main computational steps are performed and with what parameters. This helps the analyst to understand what happens to the data and how to interpret the results. Scalability implies that the workflow should cope with very large datasets, e.g. when analyzing genome-wide DNA variants or methylation patterns. To address this, it should be possible to deploy the workflow on highperformance computer resources. Reproducibility implies that the workflow will generate the same results, given the same input data, when the analysis is rerun. Many results in the biomedical literature cannot be reproduced [48] and a major difficulty behind this is differences in the local execution environment, e.g. software dependencies and package versions [49]. Tracking of provenance is related to reproducibility and requires capture of information on what software packages have been used, versions, parameters and data produced during workflow execution [27, 50].

With the miodin package, users can build streamlined analysis workflows that address the aforementioned concerns. Transparency is achieved with a clean syntax where the user only needs to specify the main steps as workflow modules, along with any necessary parameters. This makes analysis script much shorter compared to when the same analysis is implemented from scratch, 


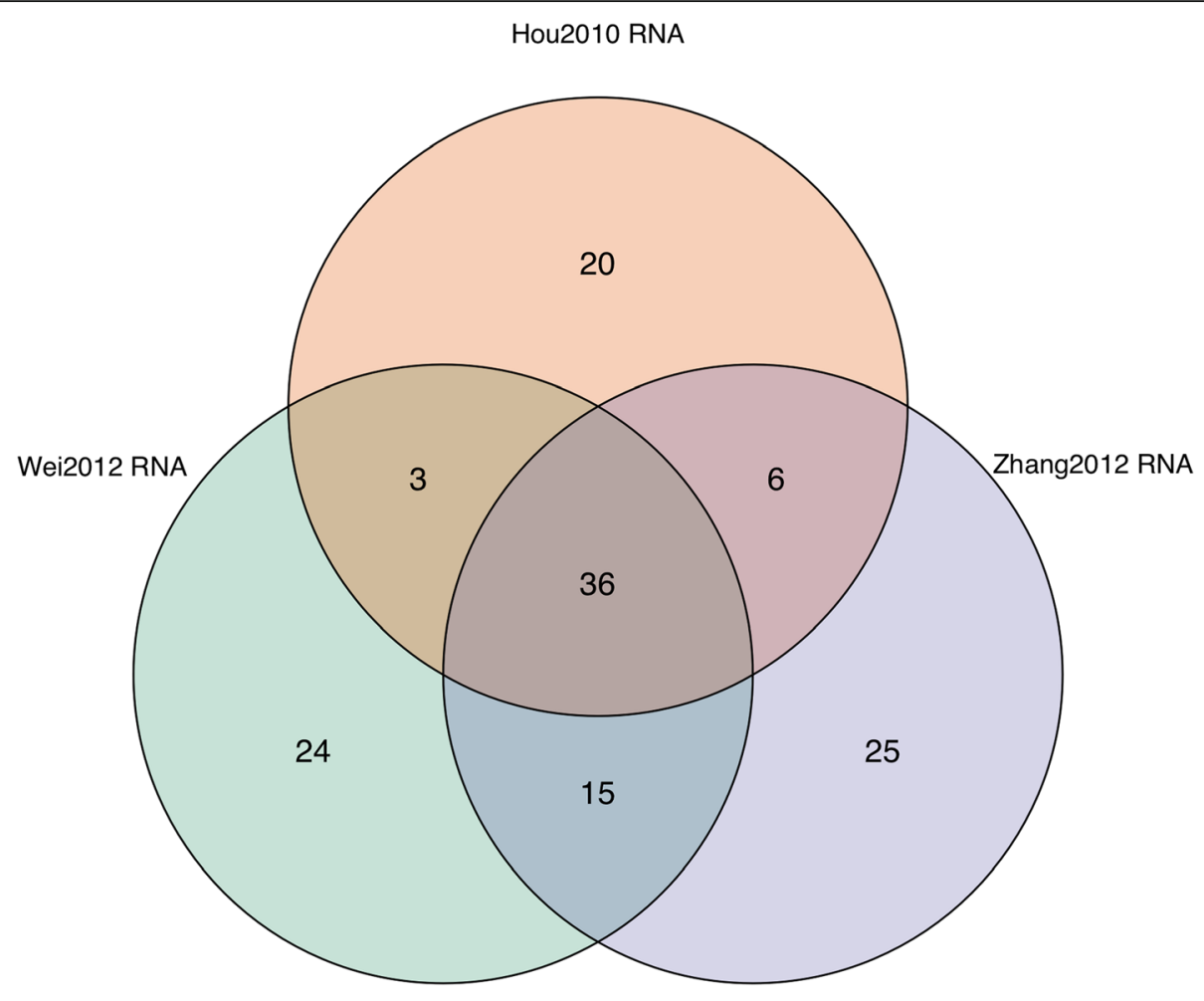

Fig. 3 Venn diagram of the number of genes identified as differentially expressed in the Wei2012, Hou2010 and Zhang 2012 datasets

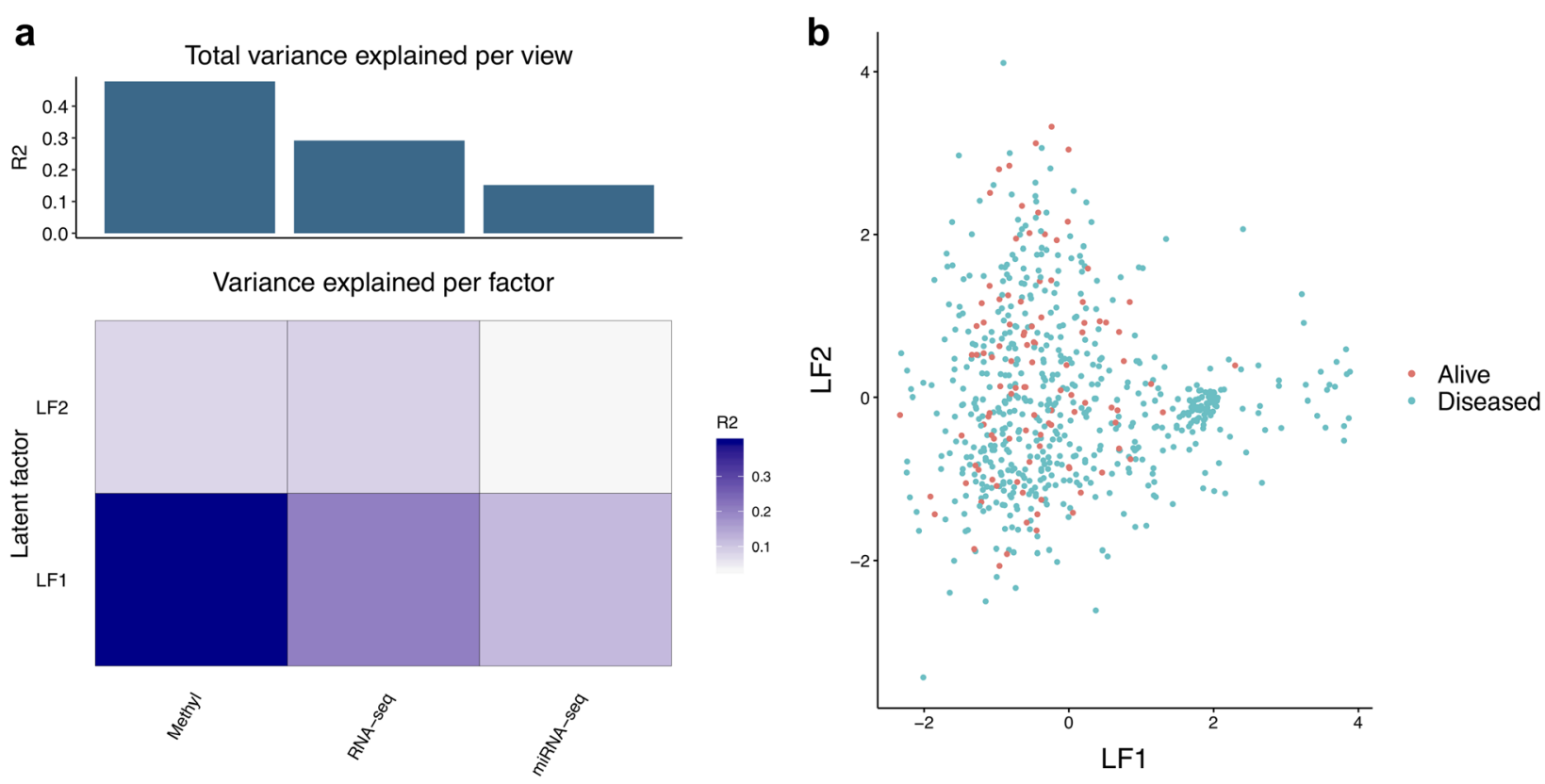

Fig. 4 Assessment of the fitted MOFA model. a shows the total amount of variance explained by the model in each omics modality (view) and variance explained per factor. $\mathbf{b}$ shows a sample ordination plot based on latent factor (LF) 1 and 2 
a
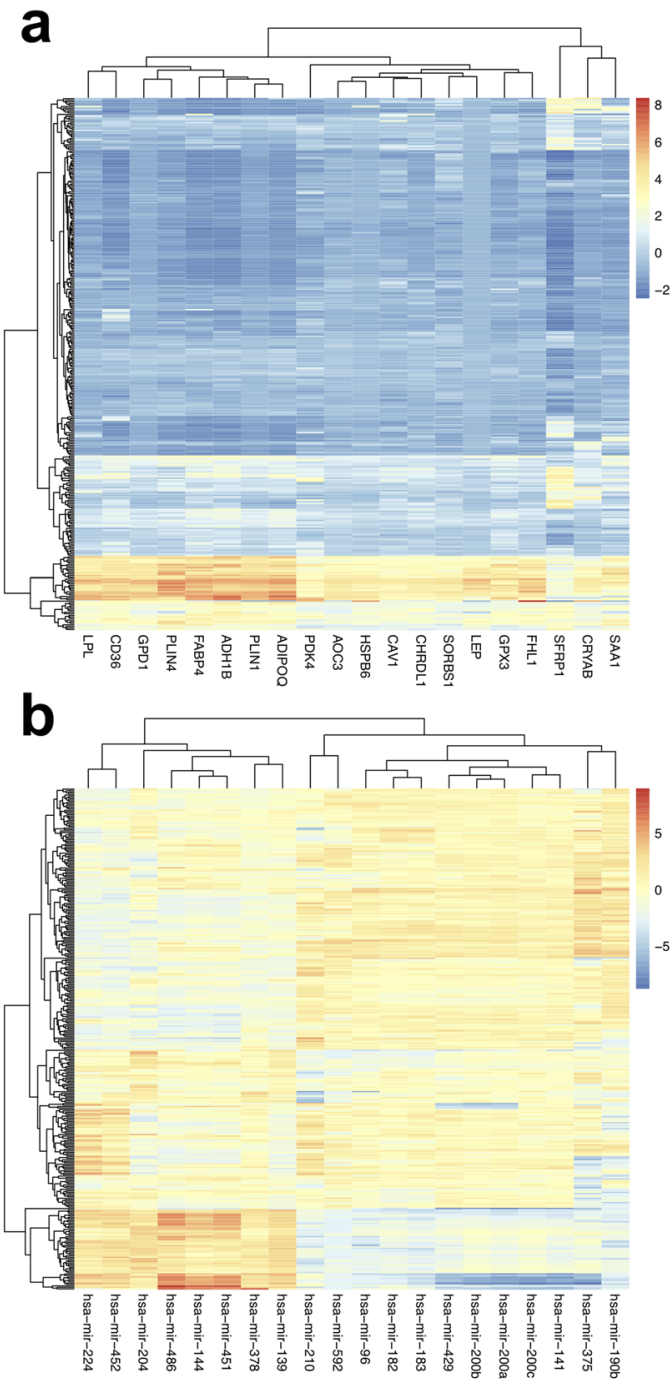

C

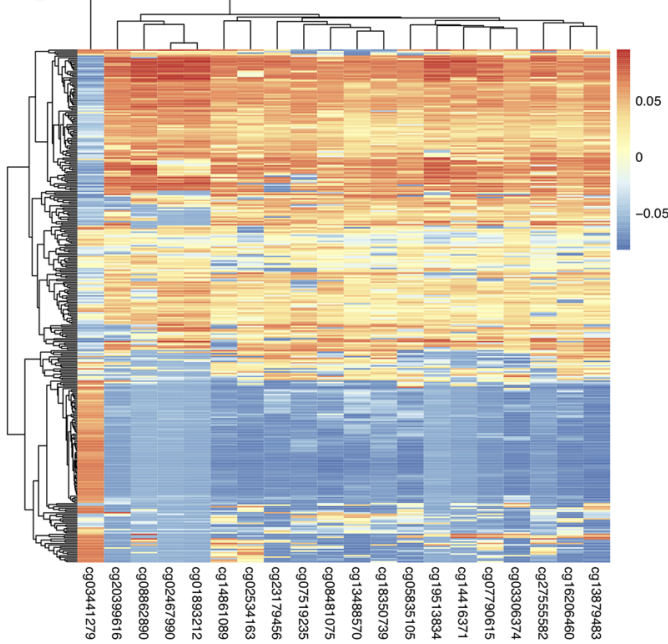

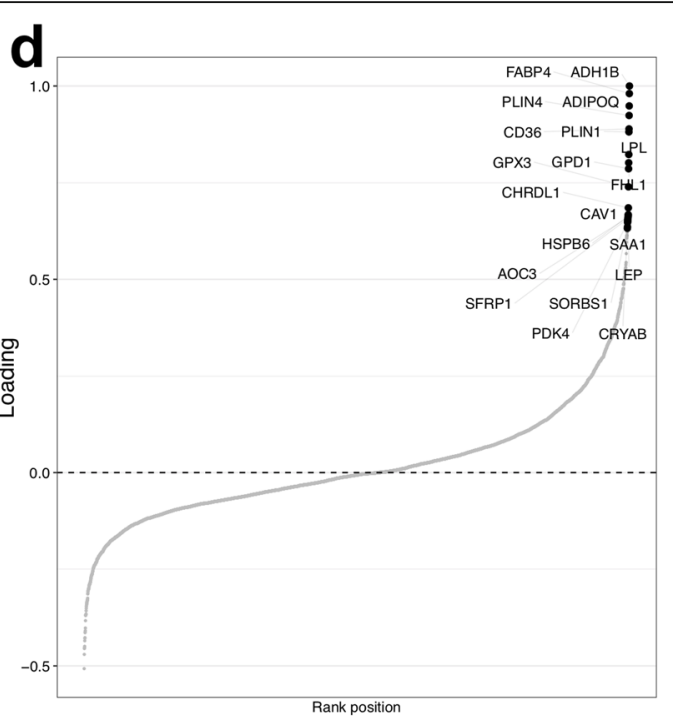

e
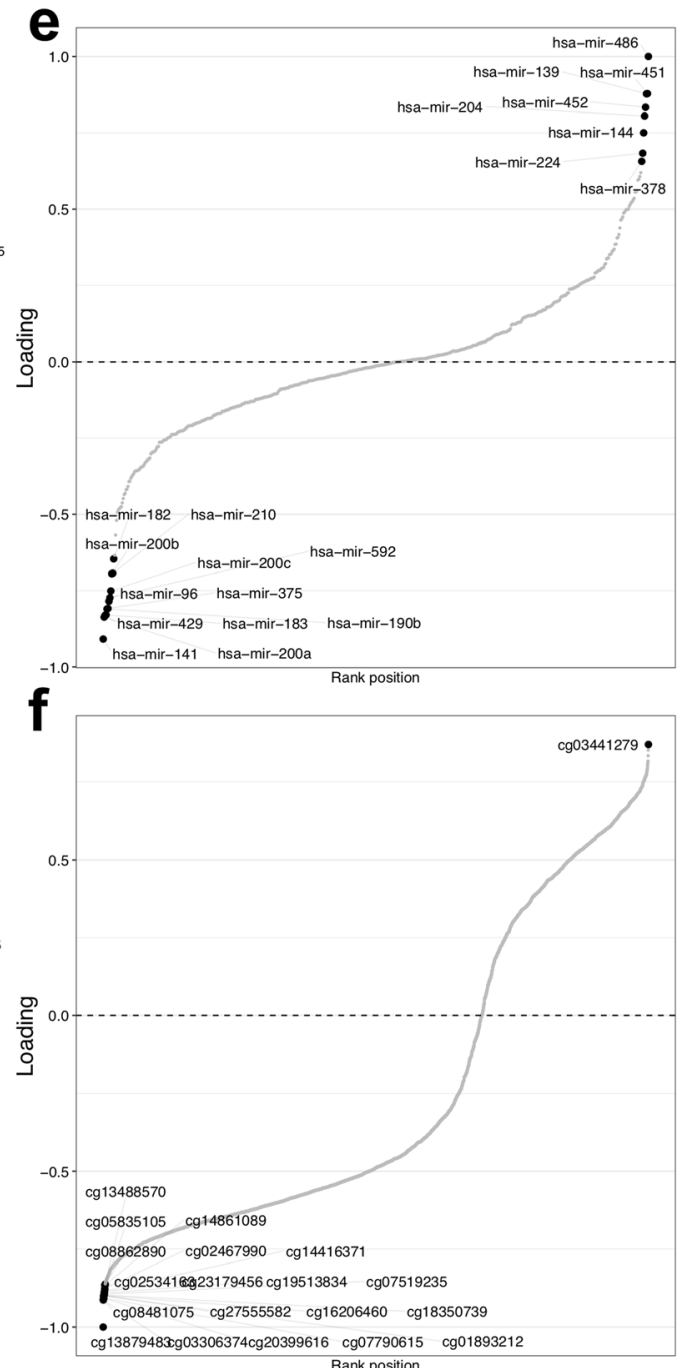

Fig. 5 Interpretation of MOFA factor 1. a through $\mathbf{c}$ show sample heatmaps with the top features in the factor for RNA-seg gene, miRNA and $450 \mathrm{k}$ methylation data, respectively. $\mathbf{d}$ through $\mathbf{f}$ reveal the loadings of the top features corresponding to the heatmaps in $\mathbf{a}$ through $\mathbf{c}$ 
since the necessary programming logic is wrapped in the modules. The steps of the workflow become visible to the analyst without having to interpret every command in a large chunk of code. Scalability is supported by translating a miodin workflow into a Nextflow [29] script, which can be deployed as a job on high-performance computer clusters. This is enabled by simply setting deployAsJob $=$ TRUE when calling execute on the workflow, provided that Nextflow is installed (see the user manual for details). Job progress can be monitored in $\mathrm{R}$ with jobStatus and the workflow configured to notify the user by email when the job is finished.

To ensure workflow reproducibility, a Docker image called miodin-notebook was configured and uploaded to DockerHub. The image can be downloaded and used to run miodin locally or when deploying workflows as jobs. Nextflow supports Docker, so the user only needs to specify the name of the image with jobContainer when executing the workflow. By running the analysis within a preconfigured container, the software environment remains constant across different systems and ensures that results can be reproduced exactly. This makes it simple to externally verify the results without spending time on configuring dependencies. Tracking data provenance also becomes easy with miodin, since the workflow modules that import, process and analyze data will automatically add steps to the dataset's processing protocol. These can be inspected in $\mathrm{R}$ or exported to Excel sheets by calling export on the dataset. This helps the analyst understand how processing steps have affected the data and to adjust parameters if necessary, prior to downstream analysis.

Several future developments are planned to enhance the functionality of the miodin package. Firstly, additional omics data types and platforms (e.g. raw sequencing and proteomics data, metabolomics, single cell, qPCR) will be supported. Secondly, several statistical and high-level analysis methods (e.g. clustering, classification, networks, annotation enrichment) will be implemented. Thirdly, workflow modules will be added for obtaining data from additional public repositories for omics, interaction and annotation data.

\section{Conclusions}

This paper presented the miodin package, which provides an infrastructure for integration and analysis of multiomics data. Key features include a high-level user API, an expressive vocabulary for declaring study designs, streamlined workflows and support for multiple omics data types and platforms. The package has been designed to promote transparent data analysis and supports scalability, reproducibility and tracking provenance during workflow execution. Jupyter notebooks are available online and can also be executed on Binder, which provides an accessible webbased interface for developing and testing workflows. To ensure the research community benefits from miodin, the software package with extensive documentation is made freely available on GitLab under the GPL-3 license.

\section{Availability and requirements \\ Project name: miodin}

Project home page: https:/gitlab.com/algoromics/ miodin

Operating system(s): Windows, Linux, MacOS

Programming language: $R$

Other requirements: Python

License: GNU General Public License v3.0

Any restrictions to use by non-academics: No

\section{Supplementary information}

Supplementary information accompanies this paper at https://doi.org/10. 1186/s12859-019-3224-4.

Additional file 1. Horizontal integration analysis script. R script for performing horizontal integration as presented in the paper.

Additional file 2. Differentially expressed genes from meta-analysis. List of genes found differentially expressed in horizontal integration analysis.

Additional file 3. Vertical integration analysis script. $\mathrm{R}$ script for performing vertical integration as presented in the paper.

\section{Abbreviations}

HCC: Hepatocellular carcinoma; LF: Latent factor; MOFA: Multi-omics factor analysis; TCGA: The Cancer Genome Atlas

\section{Acknowledgements \\ Preparation of data for vertical integration was performed on resources provided by the Swedish National Infrastructure for Computing (SNIC) at Uppsala Multidisciplinary Center for Advanced Computational Science (UPPMAX).}

Authors' contributions

The author read and approved the final manuscript.

\section{Funding}

This work has been supported by the Knowledge Foundation [grant number 20160293 and 20170302] and the Systems Biology Research Centre at University of Skövde, Skövde, Sweden. Funders had no role in the development of the software, generation of results or writing of the manuscript.

\section{Availability of data and materials}

Source code and user manual for the miodin package are available on GitLab (https://gitlab.com/algoromics/miodin). Additional file 1 contains the analysis script for horizontal integration. Additional file 2 contains the list of differentially expressed genes identified in horizontal integration analysis.

Additional file 3 contains the analysis script for vertical integration. Processed datasets used for analysis are available as part of the miodindata companion package, also available on GitLab (https://gitlab.com/algoromics/miodindata). Source datasets for horizontal integration are available from ArrayExpress with accession numbers E-GEOD-27262, E-GEOD-19188 and E-GEOD-40791. Source datasets for vertical integration are available in the curatedTCGAData package from Bioconductor, https://doi.org/10.18129/B9.bioc. curatedTCGAData.

Ethics approval and consent to participate Not applicable.

Consent for publication

Not applicable. 


\section{Competing interests}

The author declares that he has no competing interests.

Received: 14 March 2019 Accepted: 14 November 2019 Published online: 10 December 2019

\section{References}

1. Joyce $A R$, Palsson $B \varnothing$. The model organism as a system: integrating "omics" data sets. Nat Rev Mol Cell Biol. 2006;7(3):198-210.

2. Ebrahim A, Brunk E, Tan J, O'Brien EJ, Kim D, Szubin R, et al. Multi-omic data integration enables discovery of hidden biological regularities. Nat Commun. 2016;7:1-9.

3. Berger B, Peng J, Singh M. Computational solutions for omics data. Nat Rev Genet. 2013;8(9):1385-95.

4. Karczewski KJ, Snyder MP. Integrative omics for health and disease. Nat Rev Genet. 2018;19(5):29-39.

5. Woo HG, Choi JH, Yoon S, Jee BA, Cho EJ, Lee JH, et al. Integrative analysis of genomic and epigenomic regulation of the transcriptome in liver cancer. Nat Commun. 2017:8(1):839.

6. Zhu B, Song N, Shen R, Arora A, Machiela MJ, Song L, et al. Integrating clinical and multiple Omics data for prognostic assessment across human cancers. Sci Rep. 2017;7(1):1-13.

7. Lau E, Cao Q, Lam MPY, Wang J, Ng DCM, Bleakley BJ, et al. Integrated omics dissection of proteome dynamics during cardiac remodeling. Nat Commun. 2018;9(1):120.

8. Reich M, Liefeld T, Gould J, Lerner J, Tamayo P, Mesirov JP. GenePattern 2.0. Nat Genet. 2006;38(5):500.

9. Fisch KM, Meißner T, Gioia L, Ducom JC, Carland TM, Loguercio S, et al. Omics pipe: a community-based framework for reproducible multi-omics data analysis. Bioinformatics. 2015;31(11):1724-8.

10. Afgan E, Baker D, Batut B, Van Den Beek M, Bouvier D, Čech M, et al. The galaxy platform for accessible, reproducible and collaborative biomedical analyses: 2018 update. Nucleic Acids Res. 2018;46(W1):W537-44.

11. Huber W, Carey VJ, Gentleman R, Anders S, Carlson M, Carvalho BS, et al. Orchestrating high-throughput genomic analysis with bioconductor. Nat Methods. 2015;12(2):115-21.

12. Carvalho BS, Irizarry RA. A framework for oligonucleotide microarray preprocessing. Bioinformatics. 2010;26(19):2363-7.

13. Aryee MJ, Jaffe AE, Corrada-Bravo H, Ladd-Acosta C, Feinberg AP, Hansen KD, et al. Minfi: a flexible and comprehensive bioconductor package for the analysis of Infinium DNA methylation microarrays. Bioinformatics. 2014;30(10):1363-9.

14. Dvinge $H$, Bertone P. HTqPCR: high-throughput analysis and visualization of quantitative real-time PCR data in R. Bioinformatics. 2009;25(24):3325-6.

15. Gatto L, Lilley KS. MSnbase-an R/bioconductor package for isobaric tagged mass spectrometry data visualization, processing and quantitation. Bioinformatics. 2012;28(2):288-9.

16. Hahne F, LeMeur N, Brinkman RR, Ellis B, Haaland P, Sarkar D, et al. flowCore: a Bioconductor package for high throughput flow cytometry. BMC Bioinformatics. 2009;10(1):106.

17. Lawrence M, Gentleman R. VariantTools: an extensible framework for developing and testing variant callers. Bioinformatics. 2017;33(20):3311-3.

18. Liao Y, Smyth GK, Shi W. The subread aligner: fast, accurate and scalable read mapping by seed-and-vote. Nucleic Acids Res. 2013;41(10):e108.

19. Ritchie ME, Phipson B, Wu D, Hu Y, Law CW, Shi W, et al. limma powers differential expression analyses for RNA-sequencing and microarray studies. Nucleic Acids Res. 2015:43(7):e47-e47.

20. Wehrens R, Weingart G, Mattivi F. metaMS: An open-source pipeline for GC--MS-based untargeted metabolomics. J Chromatogr B. 2014;966:109-16.

21. Gentleman R. Annotate: Annotation for microarrays. R package version 1.56; 2016. p. 1.

22. Leek JT, Johnson WE, Parker HS, Jaffe AE, Storey JD. The sva package for removing batch effects and other unwanted variation in high-throughput experiments. Bioinformatics. 2012;28(6):882-3.

23. Morgan M, Anders S, Lawrence M, Aboyoun P, Pages H, Gentleman R. ShortRead: a bioconductor package for input, quality assessment and exploration of highthroughput sequence data. Bioinformatics. 2009;25(19):2607-8.

24. Robinson MD, McCarthy DJ, Smyth GK. edgeR: a bioconductor package for differential expression analysis of digital gene expression data. Bioinformatics. 2010;26(1):139-40

25. Falcon S, Gentleman R. Using GOstats to test gene lists for GO term association. Bioinformatics. 2007;23(2):257-8.
26. Luo W, Brouwer C. Pathview: an R/bioconductor package for pathwaybased data integration and visualization. Bioinformatics. 2013;29(14):1830-1.

27. Kanwal S, Khan FZ, Lonie A, Sinnott RO. Investigating reproducibility and tracking provenance - a genomic workflow case study. BMC Bioinformatics. 2017;18(1):1-14.

28. Kulkarni N, Alessandrì L, Panero R, Arigoni M, Olivero M, Ferrero G, et al. Reproducible bioinformatics project: A community for reproducible bioinformatics analysis pipelines. BMC Bioinformatics. 2018;19(Suppl 10):211.

29. Di Tommaso P, Chatzou M, Floden EW, Barja PP, Palumbo E, Notredame C. Nextflow enables reproducible computational workflows. Nat Biotechnol. 2017:35(4):316-9.

30. Merkel D. Docker: lightweight linux containers for consistent development and deployment. Linux J. 2014;2014(239):2.

31. Almugbel R, Hung LH, Hu J, Almutairy A, Ortogero N, Tamta $\mathrm{Y}$, et al. Reproducible bioconductor workflows using browser-based interactive notebooks and containers. J Am Med Informatics Assoc. 2018;25(1):4-12.

32. Ragan-Kelley M, Kelley K, Kluyver T. JupyterHub: deploying Jupyter notebooks for students and researchers; 2019.

33. Binder. 2019. Available from: https://mybinder.org. [cited 2019 Feb 2]

34. Kannan L, Ramos M, Re A, El-Hachem N, Safikhani Z, Gendoo DMA, et al. Public data and open source tools for multi-assay genomic investigation of disease. Brief Bioinform. 2016;17(4):603-15.

35. Li Y, Wu FX, Ngom A. A review on machine learning principles for multiview biological data integration. Brief Bioinform. 2018;19(2):325-40.

36. Argelaguet R, Velten B, Arnol D, Dietrich S, Zenz T, Marioni JC, et al. MultiOmics factor analysis - a framework for unsupervised integration of multiomics data sets. Mol Syst Biol. 2018;14(e8124):1-13.

37. Rohart F, Gautier B, Singh A, Lê Cao K-A. mixOmics: An R package for 'omics feature selection and multiple data integration. PLoS Comput Biol. 2017;13(11):e1005752.

38. Wang B, Mezlini AM, Demir F, Fiume M, Tu Z, Brudno M, et al. Similarity network fusion for aggregating data types on a genomic scale. Nat Methods. 2014;11(3):333-7.

39. Nguyen $T$, Tagett $R$, Diaz D, Draghici S. A novel approach for data integration and disease subtyping. Genome Res. 2017;27(12):2025-39.

40. Hernandez-Ferrer C, Ruiz-Arenas C, Beltran-Gomila A, González JR. MultiDataSet: an $\mathrm{R}$ package for encapsulating multiple data sets with application to omic data integration. BMC Bioinformatics. 2017;18(1):36.

41. Wei TYW, Juan CC, Hisa JY, Su LJ, Lee YCG, Chou HY, et al. Protein arginine methyltransferase 5 is a potential oncoprotein that upregulates G1 cyclins/ cyclin-dependent kinases and the phosphoinositide 3-kinase/AKT signaling cascade. Cancer Sci. 2012;103(9):1640-50.

42. Hou J, Aerts J, den Hamer B, van ljcken W, den Bakker M, Riegman P, et al. Gene expression-based classification of non-small cell lung carcinomas and survival prediction. PLoS One. 2010;5(4):e10312.

43. Zhang Y, Foreman O, Wigle DA, Kosari F, Vasmatzis G, Salisbury JL, et al. USP44 regulates centrosome positioning to prevent aneuploidy and suppress tumorigenesis. J Clin Invest. 2012;122(12):4362-74.

44. Parkinson H, Kapushesky M, Shojatalab M, Abeygunawardena N, Coulson R, Farne A, et al. ArrayExpress - a public database of microarray experiments and gene expression profiles. Nucleic Acids Res. 2007;35(Database issue):D747-50.

45. Ramos M, Waldron L, Schiffer L, Obenchain V, Martin M. curatedTCGAData: Curated Data From The Cancer Genome Atlas (TCGA) as MultiAssayExperiment Objects. R Packag version 120; 2018.

46. Love Ml, Huber W, Anders S. Moderated estimation of fold change and dispersion for RNA-seq data with DESeq2. Genome Biol. 2014;15(12):1-21.

47. Peters TJ, Buckley MJ, Statham AL, Pidsley R, Samaras KV, Lord R, et al. De novo identification of differentially methylated regions in the human genome. Epigenetics Chromatin. 2015;8(1):1-16.

48. Ioannidis JPA, Allison DB, Ball CA, Coulibaly I, Cui X, Culhane AC, et al. Repeatability of published microarray gene expression analyses. Nat Genet. 2009:41:149.

49. Beaulieu-Jones BK, Greene CS. Reproducibility of computational workflows is automated using continuous analysis. Nat Biotechnol. 2017;35(4):342-6.

50. Davidson SB, Freire J. Provenance and Scientific Workflows: Challenges and Opportunities. In: Proceedings of the 2008 ACM SIGMOD International Conference on Management of Data. New YorkACM; 2008. p. 1345-1350. (SIGMOD '08).

\section{Publisher's Note}

Springer Nature remains neutral with regard to jurisdictional claims in published maps and institutional affiliations. 